\title{
Unilateral linear capillaritis: A case and summary of the literature 25 years on from its original description
}

\author{
Noha Elshimy* and Benjamin Walker \\ Department of Dermatology, Harrogate District Hospital, United Kingdom
}

\begin{abstract}
Background: Unilateral Linear capillaritis is a rare but increasingly recognised subtype of pigmented purpuric dermatoses (PPD). This variant was first described in 1992 by Riordan et al and is a benign capillaritis. Its striking clinical appearance following a loose dermatomal distribution on one limb aids clinical diagnosis. We present a recent case and up to date summary of the literature with respect to this rare condition to aid the clinician in its recognition and management.

Main observation: A 56 year old lady presented with a four month history of a new, non-pruritic rash which started on the posterior left lower leg and ascended towards her buttocks. On examination she had a well-defined hyper pigmented, non-blanching, macular rash affecting the posterior left lower limb from the ankle to buttock. The linear distribution followed a S1/S2 dermatomal distribution. A punch biopsy from the left calf revealed erythrocyte extravasation, a mild dermal lymphocytic infiltrate and hemosiderin deposition; all in keeping with a rare form of pigmented purpuric dermatoses. No active treatment was commenced and 6 months later significant improvement was noted.
\end{abstract}

Conclusion: Unilateral linear capillaritis is a benign condition with a tendency to regress spontaneously over several months to a few years. No active treatment is required as patients are largely asymptomatic. This is a key factor for clinicians to be aware of as the summary of the literature does show that patients have been exposed to treatments which are largely ineffective and may expose the patient to more risk than benefit.

\section{Introduction}

We present a case of Unilateral Linear Capillaritis; a rare form of pigmented purpuric dermatoses. This variant was first described by Riordan et al and published in 1992 in Clinical and Experimental Dermatology [1]. We now present a case 25 years after it was originally described whilst noting there are only 14 other cases reported in the literature; the findings of which we have summarised (Table 1). This clearly is a rare condition of which we hope to summarise the key learning points.

\section{Case report}

A 56 year old lady presented to the dermatology department with a one month history of a new, non-pruritic rash on the left posterior lower limb. The primary concern was the aesthetic appearance rather than any other associated symptoms as she had otherwise been completely asymptomatic. She is known to have type 2 diabetes mellitus and hypercholesterolemia. Her regular medications are aspirin, simvastatin, metformin, losartan and dapagliflozin. There had been no recent medication changes and no over the counter or herbal remedy usage.

On Examination, she had a striking linear rash extending from the left posterior ankle to the left buttock. This consisted of nonblanching, hyper-pigmented scattered macules loosely fitting an S1/ S2 dermatomal distribution (Figure 1). The skin on her right leg was completely normal. At this point the clinical diagnosis was felt to be unilateral linear capillaritis. The patient went on to have a $4 \mathrm{~mm}$ punch biopsy from the left calf and also from the left posterior thigh. This revealed evidence of a mild dermal lymphocytic infiltrate and evidence of erythrocyte extravasation was also noted. A Perl's stain was positive indicating hemosiderin deposition (Figure 2). These are the key histological features found in unilateral capillaritis.
As the patient was asymptomatic, a conservative approach was taken towards her management with no active treatment commenced. She was followed up twice during the following six months. On each visit, it was noted that the rash was fading and that there did appear to be areas of regression. She remained asymptomatic throughout.

\section{Discussion}

Pigmented purpuric dermatoses (PPD) is an umbrella term used to describe an often asymptomatic eruption of purpuric lesions with similar histological features that essentially are all in keeping with a benign capillaritis [1]. The aetiology remains unclear hence why there is no clear consensus on treatment options. The original description of PPD included five main subtypes namely; Schamberg's purpura, Majocchi purpura, lichen aureus, pigmented purpuric lichenoid dermatosis of Gougerot and Blum and eczematis-like purpura of Doucas and Kapetanakis [2].

Schamberg's purpura often presents with pigmented patches or macules with well-defined areas of punctate purpura which are often classically described as 'cayenne pepper' spots [1]. The distribution is usually symmetrical on the lower limbs of middle ages adults and most patients are largely asymptomatic [1]. Meanwhile, Majocchi's purpura (also known as purpura annularis telangiectodes) is more commonly

Correspondence to: Noha Elshimy, Department of Dermatology, Harrogate District Hospital, United Kingdom, Tel: 07539735222; E-mail: noha@doctors. org.uk

Key words: pigmented purpuric dermatoses, mild dermal lymphocytic infiltrate, benign capillaritis, adolescents, hemosiderin deposition

Received: June 07, 2017; Accepted: June 26, 2017; Published: June 28, 2017 
Table 1. Table summarising the literature and should be titled: 'A summary of the literature to date.

\begin{tabular}{|c|c|c|c|c|c|}
\hline Author & Site & Age & Gender & Treatment & Outcome \\
\hline Riordan et al. [1] & Left lower leg & 13 & Male & Nil & Spontaneous regression 30 months later \\
\hline Riordan et al. [1] & Right lower leg & 13 & Male & Nil & Significant regression 14 months later \\
\hline Riordan et al. [1] & Right lower leg & 25 & Male & Clobetasol Propionate-no improvement & Complete resolution 3 years later \\
\hline Riordan et al. [1] & Left lower leg & 38 & Male & Nil & Eruption fading at 18 months \\
\hline Ma et al. [5] & Right upper limb & 29 & Male & Nil given for capillaritis & Spontaneous regression at 20 months \\
\hline Ma et al. [5] & Left upper limb & 23 & $\begin{array}{l}\text { Female (arose in } \\
\text { pregnancy) }\end{array}$ & $\begin{array}{l}4 \text { weeks oral hydroxchloroquine and topical } \\
\text { steroid-no improvement. } \\
2 \text { months of PUVA with some improvement. }\end{array}$ & Improvement with PUVA \\
\hline Hersh CS et al. [6] & Left lower limb & 15 & Male & Unclear & Unclear \\
\hline Mar A et al. [7] & $\begin{array}{l}\text { Left lower lower limb and } \\
\text { left torso }\end{array}$ & 8 & Male & Betamethasone $0.05 \%$ for 2 months & $\begin{array}{l}\text { Purpura resolved at } 3 \text { months, pigmentation } \\
\text { partially faded at } 12 \text { months }\end{array}$ \\
\hline Mar et al. [7] & Left abdomen & 12 & Female & Mometasone Furoate $0.1 \%$ for 6 weeks & $\begin{array}{l}\text { Purpura resolved at } 6 \text { weeks, pigmentation partially } \\
\text { faded at } 12 \text { months }\end{array}$ \\
\hline Mar et al. [7] & Left posterior thigh & 7 & Male & Mometasone Furoate $0.1 \%$ for 3 months & Purpura resolved at 3 months follow up \\
\hline Higgins et al. [8] & $\begin{array}{l}\text { Right lower limb and right } \\
\text { lower trunk }\end{array}$ & 48 & Female & Nil & Spontaneous regression over several months \\
\hline \multirow[t]{2}{*}{ Taketuchi et al. [9] } & Left upper limb & 35 & Female & Topical steroid-unclear potency & Unclear \\
\hline & & & & 'slight effect' & Lost to follow up \\
\hline Taketuchi et al. [9] & Right lower limb & 26 & $\begin{array}{l}\text { Female } \\
\text { Aspirin induced }\end{array}$ & $\begin{array}{l}\text { Potent topical steroid Oral Vitamin C } \\
\text { Tranexamic Acid } \\
\text { None led to any improvement }\end{array}$ & $\begin{array}{l}\text { Stopped Aspirin } \\
\text { Disappeared 2-3 months later }\end{array}$ \\
\hline Pock et al. [10] & Right upper limb & 5 & Female & Nil & Spontaneous resolution at 18 months \\
\hline
\end{tabular}

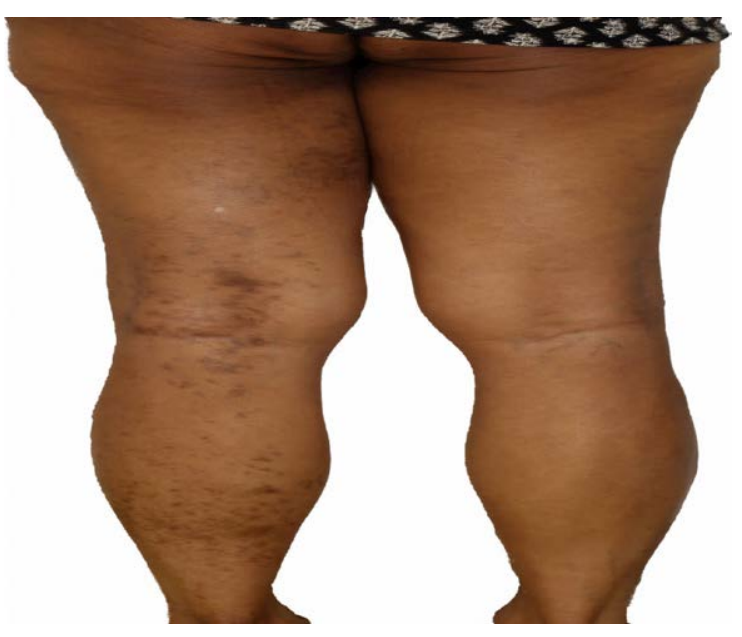

Figure 1. Clinical Photograph demonstrating loose dermatomal distribution of rash.

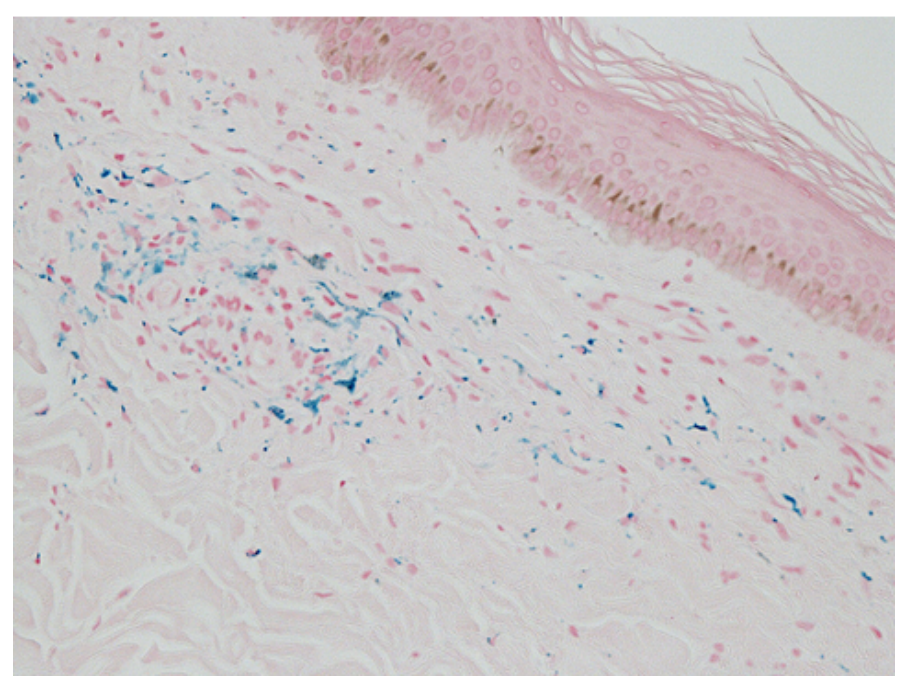

Figure 2. Histology slide revealing positive staining with Perls' Prussian blue indication of hemosiderin deposition. reported in adolescents and young adults presenting with annular patches of punctate purpura with surrounding telangiectasia in a symmetrical distribution on the lower limbs [1-3]. A chronic localised variant of PPD is Lichen aureus, which most commonly presents on a lower limb in a unilateral distribution with golden- brown papules [1]. In pigmented purpuric lichenoid dermatosis of Gougerot and Blum, patients tend to present with red-orange lesions and purpuric plaques that can be associated with erythema and pruritis [1].Furthermore, it has been reported to take on a chronic course of up to two years [1]. Finally, in eczematis-like purpura of Doucas and Kapetanakis (also known as itching purpura) the lesions do resemble those noted in Schamberg's disease however it may take on a more widespread distribution and involve the lower body [1]. Clinically, scaling and epidermal change are often noted and patients do present with pruritus, hence the name [1].

It has however become increasingly apparent that there are variants which do not classically fit one of these five subtypes, including granulomatous variants and unilateral linear capillaritis [4]. The striking linear appearance of what are often purpuric lesions in unilateral linear capillaritis is a key clinical feature that separates it from other subtypes which tend to take on a symmetrical distribution. We believe we have provided the most recent summary of the literature (Table 1).

Although it is difficult to draw conclusions regarding epidemiology and treatment outcome due to the rarity of the condition; the findings in Table 1 do suggest that lower limbs are more commonly involved compared to upper limbs and that unilateral linear capillaritis affects a younger demographic of patients with the age range of 5-48 years. The majority of patients were not actively treated and most patients seemed to notice spontaneous regression over months, with a maximum of 30 months noted in one report.

Our summarised data does in fact reinforce that unilateral linear capillaritis is a benign condition with a tendency to spontaneously regress. Therefore it seems unlikely that any active treatment is actually required. Furthermore, treatments such as potent topical steroids, hydroxychloroquine and PUVA which were offered in a few cases may in fact be associated with a greater risk profile to the patient, especially as this is usually an asymptomatic condition. 


\section{Learning points}

1. Unilateral linear Capillaritis is a rare subtype of the pigmented purpuric dermatoses.

2. The rash can affect upper or lower limbs but tends to consist of hyper-pigmented, purpuric macular lesions that take on a striking linear distribution on one limb; hence the name.

3. Patients are usually asymptomatic and present due to the aesthetic appearance.

4. The key histological features include a mild-moderate dermal lymphocytic infiltrate, erythrocyte extravasation and hemosiderin deposition.

5. Unilateral linear capillaritis is benign and the natural history of this condition is that it will resolve spontaneously over several months to a few years.

6. There is insufficient evidence to recommend a particular treatment option especially as most patients are asymptomatic. Some patients may be subjected to unnecessary treatments which in themselves have their own side effect profiles.

\section{References}

1. Riordan CA, Darley C, Markey AC, Murphy G, Wilkinson JD (1992) Unilateral linear capillaritis. Clin Exp Dermatol 17: 182-185. [Crossref]

2. Zalaudek I, Ferrara G, Brongo, Giorgio CM, Argenziano G (2006) Atypical clinical presentation of pigmented purpuric dermatosis. J Disch Dermatol Ges 4: 138-140. [Crossref]

3. Sardana K, Sarkar R, Sehgal VN (2004) Pigmented purpuric dermatoses: an overview. Int $J$ Dermatol 43: 482-488. [Crossref]

4. Wong WR, Kuo TT, Chen MJ, Chan HL (2001) Granulomatous variant of chronic pigmented purpuric dermatosis: report of two cases. Br J Dermatol 145: 162-164. [Crossref]

5. Ma HJ, Zhao G, Liu W, Dang YP, Li DG (2007) Unilateral linear capillaritis: two unusual Chinese cases. Eur J Dermatol 17: 160-163. [Crossref]

6. Hersh CS, Shwayder TA (1991) Unilateral progressive pigmentary purpura (Schamberg's disease) in a 15-year-old boy. J Am Acad Dermatol 24: 651. [Crossref]

7. Mar A, Fergin P, Hogan P (1999) Unilateral pigmented purpuric eruption. Australas $J$ Dermatol 40: 211-214. [Crossref]

8. Higgins EM, Cox NH (1990) A case of quadrantic capillaropathy. Dermatologica 180: 93-95. [Crossref]

9. Taketuchi Y, Chinen T, Ichikawa Y, Ito M (2001) Two cases of unilateral pigmented purpuric dermatosis. J Dermatol 28: 493-498. [Crossref]

10. Pock L, Capkova S (2002) Segmental Pigmented Purpura. Paediatr Dermatol 19: 517519. [Crossref]

Copyright: (C2017 Elshimy N. This is an open-access article distributed under the terms of the Creative Commons Attribution License, which permits unrestricted use, distribution, and reproduction in any medium, provided the original author and source are credited. 